\title{
Telemedicine in the acute care setting during the COVID-19 pandemic
}

\author{
Ziwei Lin ${ }^{1,2}$ - Tiong Beng Sim ${ }^{1,2,3} \cdot$ Victor Yeok Kein Ong ${ }^{1,2,3} \cdot$ Zulkarnain bin Ab Hamid $^{1,2,3} \cdot$ Weng Hoe Ho ${ }^{1,2,3}$
}

Received: 7 April 2020 / Accepted: 20 July 2020 / Published online: 3 August 2020

(C) Società Italiana di Medicina Interna (SIMI) 2020

\section{Dear Editor,}

As of 10th July 2020, coronavirus disease 2019 (COVID-19) has afflicted 12,102,328 people and claimed 551,046 lives [1]. Transmission of the SARS-COV-2 virus to healthcare workers has been well documented and attributed to lack of personal protective equipment (PPE) and long duration of exposure to large numbers of infected patients [2]. Healthcare workers in the emergency department (ED) are frontline staff and are at increased risk of COVID-19 exposure and nosocomial infection. The use of telemedicine may mitigate this.

We are a team running a 24-h urgent care centre (UCC) at Alexandra Hospital, a 215-bed general hospital in Singapore. To prevent spread of COVID-19 within the centre, an extended screening area (ESA) was constructed in a wellventilated sheltered area outside the main building. The ESA caters to patients with presentations suspicious for COVID19 such as respiratory complaints, fever, or contact history that increases their risk of exposure to the virus (e.g. contact with a known COVID-19 patient, had visited an area where a significant number of patients with COVID-19 were identified, etc.).

The ESA comprises a doffing area, waiting area, triage area, consultation rooms, $\mathrm{X}$-ray room, as well as an area for nasopharyngeal swabs to be obtained (Fig. 1). The waiting area consists of plastic chairs placed a minimum distance of $2.5 \mathrm{~m}$ apart to prevent transmission between patients. It has a maximum capacity of 20 patients. Patients are first screened by a security officer equipped with full PPE and

Ziwei Lin

ziwei_lin@ nuhs.edu.sg

1 Emergency Medicine Department, National University Hospital, National University Health System, 9 Lower Kent Ridge Road, Singapore 119085, Singapore

2 Urgent Care Centre, Alexandra Hospital, National University Health System, Singapore, Singapore

3 Department of Surgery, Yong Loo Lin School of Medicine, National University of Singapore, Singapore, Singapore then directed to the ESA if they meet any of the criteria listed above. Once in the ESA, they will be triaged by the nursing staff before being assigned to a specific seat to await consultation. Seat numbers are documented for contact tracing purposes in case the patient tests positive for COVID-19. A physician will enter the ESA to take a history from the patient and examine him/her before ordering investigations. After reviewing the results and deciding the disposition, the physician then re-enters the ESA to communicate this to the patient. Terminal cleaning is done after each patient encounter in the ESA. Prior to entering the ESA, healthcare workers don full PPE, comprising an N95 respirator, face shield or goggles, surgical cap, gown, and gloves. After exiting the ESA, healthcare workers will doff their PPE and perform hand hygiene measures before entering the main building.

In April 2020, ESA attendances constituted a significant $57.6 \%$ of our total attendances $(643 / 1117)$. Every shift at the UCC is manned by one emergency medicine specialist and one to two medical officers, making it impractical to have a dedicated physician manning the ESA. Hence, physicians would repeatedly doff and don PPE to switch between the ESA and main building. This limitation, coupled with the ESA's small waiting capacity, necessitated measures to increase the efficiency of our consultations.

Our centre thus introduced the use of telemedicine via video conferencing to facilitate history taking for patients in the ESA. Internet-capable laptops were installed in the ESA and main building, allowing healthcare workers to communicate with patients via teleconferencing. This allows for history taking and visual inspection of patients. Investigations (e.g. plain radiographs, laboratory tests) and medications can then be ordered. The physician will subsequently enter the ESA to examine the patient, and a nasopharyngeal swab will be taken if needed. This workflow is only used for patients with normal vital signs without signs of distress, i.e. with a low acuity triage. Unstable or ill patients are seen directly in negative-pressure isolation rooms within the main building.

Teleconferencing allows us to conduct the history and discharge communications from within the main building, reducing the time spent in PPE and the duration of exposure 

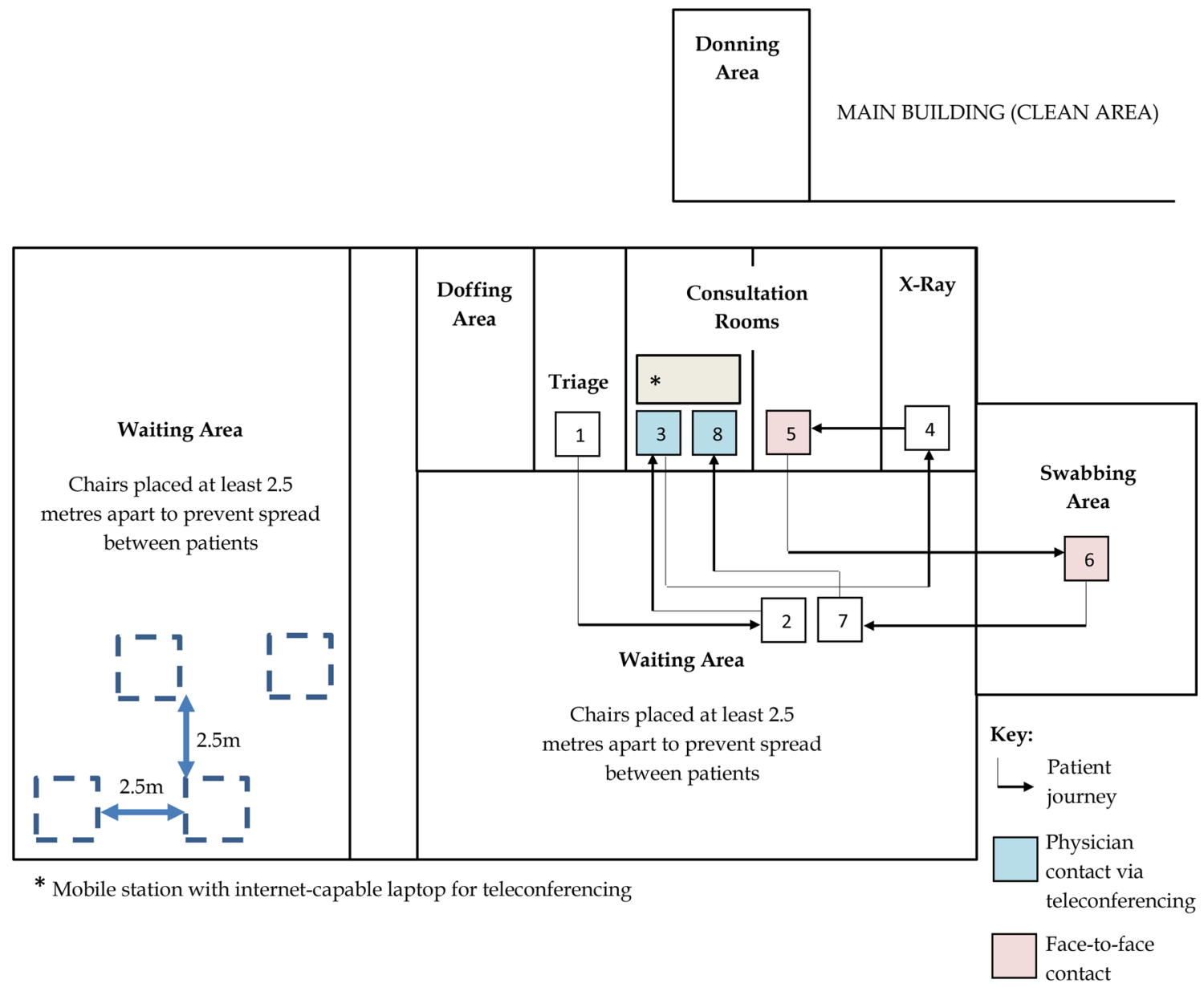

ESA workflow with use of telemedicine consultation

1. The patient is brought into the triage area in the ESA if they meet the screening criteria.

2. After being triaged, the patient is directed to the waiting area.

3. At their turn for consult, the patient enters the consult room with the mobile station where a full history and general inspection is conducted by a physician remotely via teleconferencing.

4. Investigations such as laboratory tests and X-rays are then ordered remotely and completed by the ESA nurse and radiographer respectively.

5. The physician enters the ESA after donning a full set of personal protective equipment (PPE) and conducts a physical examination for the patient.

6. A nasopharyngeal swab test for SARS-CoV-2 is also done by the physician at this time if indicated.

7. The patient returns to his or her designated seat in the waiting area while awaiting results of the investigations.

8. After review of results, the management plan is communicated and discussed with the patient via teleconferencing again. The patient is then either discharged from the ESA or admitted to an isolation ward.

Fig. 1 Diagram showing layout of the extended screening area (ESA)

to infectious patients. The physician is only required to enter the ESA to conduct a physical examination and obtain a nasopharyngeal swab. Obtaining a nasopharyngeal swab takes less than a minute to perform. Telemedicine is thus useful where history taking may form bulk of the consult, such as in an infectious disease outbreak, where the physician needs to delve into details that will ascertain exposure to the disease. This includes details such as the patient's occupation, contact history, travel history, and whether he/ she may have been to areas where clusters of patients with the infection have previously been identified. An audit done showed that the time spent in the ESA was reduced with the use of telemedicine $(p=0.002)$, from a mean of $20.2 \mathrm{~min}$ (standard deviation [SD] $7.8 \mathrm{~min}$ ) per patient without the 
use of teleconferencing $(n=9)$, to $8.4 \mathrm{~min}(\mathrm{SD} 2.6 \mathrm{~min})$ per patient while teleconferencing was used $(n=9)$. This affords flexibility in manpower allocation, allowing healthcare workers who may be unsuitable to see suspect cases (e.g. if the healthcare worker is immunocompromised) to assist with history taking and ordering of investigations or medications of multiple patients in the ESA simultaneously.

Telemedicine is often used when patients are in remote locations, but can also be used on site even if all parties are present as a means of reducing time of exposure to an infectious patient. Reducing time spent in PPE may also be beneficial for healthcare workers as prolonged PPE usage is associated with increased physiological strain such as heat stress and fatigue [3]. Implemented in this way, it also helps to reduce utilization of PPE resources, which may be in short supply during pandemics.

Other uses of telemedicine in the ED would be for "forward triage", where patients can be triaged even prior to patient arrival [4]. This allows patients to be isolated or deisolated expeditiously, and resources such as isolation rooms and PPE can be utilized in a more efficient manner. In situations where healthcare staff and patients are unable to communicate due to language barriers, telemedicine may provide a platform for a translator in a three-way conversation from a remote location if translators are not available on-site. While telemedicine has been widely used for medical emergencies such as acute stroke [5], its use has not been as established in other conditions. Telemedicine may provide an avenue for other subspecialty consults in the ED if they are not available on-site, expediting care and potentially reducing the need for transfer of infectious patients.

Telemedicine has its limitations: it is unsuitable for unstable patients requiring emergent intervention, and procedures and certain physical examination manoeuvres (e.g. chest auscultation) cannot be done remotely. For cases where history -taking can be done quickly, time savings might not be as substantial. Technical difficulties and equipment failures may also make its use less efficient. Centres also may not have necessary infrastructure or protocols for its implementation and maintenance.

Despite this, telemedicine remains an invaluable resource during outbreaks, and can be more widely utilized in the emergency and acute care setting. The COVID-19 pandemic will be a long-drawn battle, and there may need to be a paradigm shift in the way telemedicine is used. Further research should be done to optimize its use, whether for the purposes of the COVID-19 outbreak, or in future infectious disease outbreaks. We hope that other centres may find this strategy useful.

Author contributions All authors were involved in the drafting and revision of the manuscript.

Funding None.

\section{Compliance with ethical standards}

Conflict of interest The authors declare that they have no conflict of interest.

Human and animal rights This study was reviewed and deemed exempt by the National Healthcare Group Domain Specific Review Board.

Informed consent Not applicable

\section{References}

1. World Health Organization (2020) Coronavirus disease 2019 (COVID-19) Situation Report-172. https://www.who.int/docs/ default-source/coronaviruse/situation-reports/20200710-covid-19sitrep-172.pdf?sfvrsn=70724b90_2. Accessed 11 Jul 2020

2. Wang J, Zhou M, Liu F (2020) Reasons for healthcare workers becoming infected with novel coronavirus disease 2019 (COVID-19) in China. J Hosp Infect 105:100-101. https://doi. org/10.1016/j.jhin.2020.03.002

3. Grélot L, Koulibaly F, Maugey N et al (2016) Moderate thermal strain in healthcare workers wearing personal protective equipment during treatment and care activities in the context of the 2014 Ebola virus disease outbreak. J Infect Dis 213:1462-1465. https://doi.org/10.1093/infdis/jiv585

4. Hollander JE, Carr BG (2020) Virtually perfect? Telemedicine for Covid-19. N Engl J Med 382:1679-1681. https://doi.org/10.1056/ NEJMp2003539

5. Wechsler LR, Demaerschalk BM, Schwamm LH et al (2017) Telemedicine quality and outcomes in stroke: a scientific statement for healthcare professionals from the American Heart Association/American Stroke Association. Stroke 48:e3-e25. https://doi. org/10.1161/STR.0000000000000114

Publisher's Note Springer Nature remains neutral with regard to jurisdictional claims in published maps and institutional affiliations. 